\title{
THE ASSESSMENT OF THE AFFECTIVE-BEHAVIORAL DEVELOPMENT OF 2-3 YEARS OLD CHILDREN WITH AUTISM AND PERVASIVE DEVELOPMENTAL DISORDERS
}

\author{
Marina Bardyshevskaya \\ Department of Psychology, Moscow State University named after M.V. Lomonosov, Moscow (Russia)
}

\begin{abstract}
The model and method of level development of affective-behavioral complexes (ABC, Bardyshevskaya) is presented in this paper. As an illustration, the paper presents the experience of using the ABC method to identify affective behavioral development options in 2-3 year old children with autism spectrum disorders (ASD) and pervasive developmental disorders (PDD).
\end{abstract}

Keywords: Children, autism, development, assessment, affective-behavioral complexes.

\section{Introduction. The fundedness of the $\mathrm{ABC}$ model and method. Novelty and relevance}

The model of the ABC was developed by Marina Bardyshevskaya $(2012,2018)$ on the basis of generalized data from systematic participant observation of 435 children with severe affective instability (autism, drive-related and behavioral disorders, emotional disorders, psychotic states). The empirical data used to create the model and the ABC method were collected by longitudinal observation (lasting from several months to 14 years, on average 2-3 years) of the mental development of 80 children aged from 0 to 16 years old. The number of hours of observations with documented data (written records after observation and sample video recordings of data) for each child varied from 3 to 120. Observations were conducted in 1996-2018 years firstly in the children's home and then, since 1997, mainly in the Moscow Scientific and Practical Centre for Mental Health of Children and Adolescents named after G.E. Sukhareva.

The ABC method allows to construct individual affective-behavioral profiles (diagrams of the $\mathrm{ABC}$ development) of a person on the basis of the analysis of observed behavior (based on video recording of diagnostic sessions). In the course of observation, stable patterns of emotional states and behavior are identified in response to specific affective loads for each ABC. The way the transition from one type of behavior to another is carried out shows the vector (zone of the proximal development) of different behavior types. The ABC method being used in work with patients with autism allows us to get out of the rigid framework of medical diagnosis and clearly show asynchrony between the development of different types of behavior, the amplitude of oscillations: the zone of proximal development (towards the norm) and the zone of risk of irreversible changes (towards disintegration). The ABC method differentiates moderate and mild forms of autism development more precisely than CARS (Childhood Autism Rating Scale) and is more sensitive to the dynamics of mental development of a child with ADS during treatment. A comprehensive qualitative and quantitative description of the individual profile of all ABCs makes it possible to draw up a scientifically substantiated correction program.

The sources of the model are the notion of age-specific symptoms in child psychiatry (Sukhareva, 1959); classification of disonthogenesis (Lebedinsky, 1985); theory of level organization of movements (Bernstein, 1967); level model of emotional regulation (Lebedinsky et al., 1990; Bardyshevskaya, Lebedinsky, 2003); and also John Bowlby's attachment theory; Niko Tinbergen's ethological model of autism, psychoanalytic infant observation and some others.

\section{The ABC model}

The notion of an "affective-behavioural complex" (ABC) is introduced to denote holistic formations that are stable and easily recognised, which appear spontaneously during the course of maturation and development and which have become entrenched in evolution (Bardyshevskaya, 2012).

Each $\mathrm{ABC}$ includes emotional states of a specific register, which change in a particular range, specific repertoire of behavioural patterns, types of contact with people and objects and symbolic activity that correspond to them, the links between elements of one $\mathrm{ABC}$ and between different $\mathrm{ABCs}$, sensitive to a specific range of affective loads, and specific mechanisms of emotional regulation. These 
mechanisms do not allow critical emotional outbursts that may be dangerous for the development. Affective loads may be bearable, unbearable, optimal (they provide the safe movement to the zone of proximal development).

The elements of the $\mathrm{ABC}$ are emotional states, a special repertoire of eating behaviour, sleep and wakefulness, territorial, excretory, sexual and exploratory types of behaviour, attachment, aggression, non-verbal (demonstrative) communication and forms of symbolic activity: play, art-depictive (drawing, modeling), speech behavior and the behaviour in front of a mirror (14 items total), as well as certain kinds of links between elements of an ABC and between the ABC and the other ABCs (Bardyshevskaya, 2018).

There are 5 ABCs (with their sensitive periods): 1) the psychophysiological surviving $A B C$ (intrauterine period - the first 6-8 weeks), the $\mathrm{ABC}$ of symbiotic type $(0-18-24$ months), the $\mathrm{ABC}$ of expansion ( 8 months - 36-48 months), the play-dialogue and attachment ABC (3 months - 24-36 months) and a role-imaginative $\mathrm{ABC}(24$ months -5 years $)$.

It is important to note the unity of semantic and technical organization of affect, behavior, motivation, communication and symbolization in ABCs (in different activity modes). For example, manifestations of different movement organization disorders which are typical for people with affective instability are unstable and vary in severity significantly. These variations depend on the actual affective load. So, we assess any particular behaviour in different regimes of activity separately. The visualization of the observation in an umbrella diagram (see Fig. 1) looks like a kind of "wind rose" of emotional states, non-verbal communication and behavioral development.

Figure 1. An example of a complex diagram that shows the scores for the five ABCs altogether of $M$. -2 years old boy with pervasive developmental disorder (PDD).

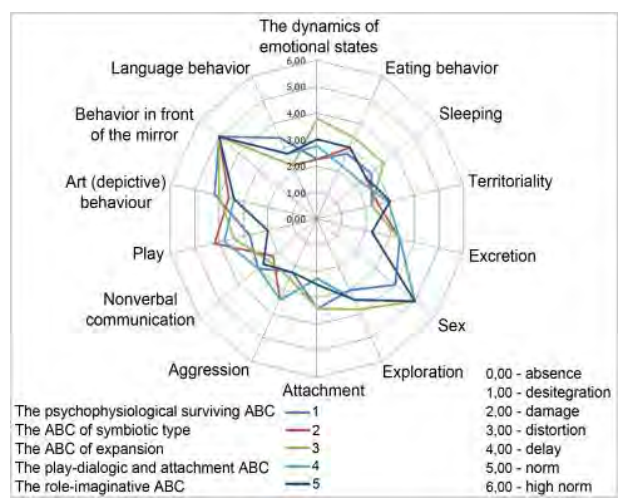

The dominant $\mathrm{ABC}$ is identified on the types of connections the person does. Rapid assessment of the dominant $\mathrm{ABC}$ is made by analyzing the child's current non-verbal communication and by assessing the connections that the child establishes between the toys. These connections are assumed to reflect the child's connections to the people as he or she perceives them at the moment.

The following types of connections are most common for 2-3 years old children with autism. The absolute dominance of the psychophysiological surviving $\mathrm{ABC}$ is characterized by: isolation disruption of any connections, blockade arising in a state of critical decline in psychic tone: fusion, dissolution, confusion, interrupted connection. Tunnel connection is a transitional form of connection between the psychophysiological surviving $\mathrm{ABC}$ and the $\mathrm{ABC}$ of expansion: in order not to be afraid of depth, the child "erases" what is around the focus of his or her attention; this includes isolation of a certain element, keeping it in a container or a "compartment". Chaos, pile, fragmentation is observed in patients with the predominant psychophysiological survival $\mathrm{ABC}$ with a tendency to transition to $\mathrm{ABC}$ of expansion (the child is not afraid of leaving traces or actively destroying things). The predominant ABC of symbiotic type is characterized by the series, chains (varying in lengths and degree of rigidity). A spider web, a network (a group without a hierarchy) is observed at the stage of a child's transitioning from the predominant $\mathrm{ABC}$ of symbiotic type to the $\mathrm{ABC}$ of expansion. More complex types of connection (hierarchy: rigid and flexible) are rarely observed in 2-3 year old children with autism. However, it is important that the psychologist working with such a child thinks about these connections as a zone of possible (although not proximal) development.

The distance from each pattern to the norm can be approximated in points, which are used to build diagrams of the ABC development (Bardyshevskaya, 2018, p. 18-21):

High Norm (6.00). The complex pattern is used steadily, appears before the "right" age. Norm (5.00). "Breakthrough" — the child enjoys practicing a pattern, it occurs at about "the right" age and is used spontaneously, steadily, without special helping effort from an adult.

Delay (4.00). Age-corresponding pattern appears on a negative emotional background, it is pronouncedly incomplete, it requires a lot of external stimulation, effort from the outside. The first unstable signs or precursors of pattern emerge. Delay towards distortion (3.60). The child attaches and 
fixates a specific meaning to the pattern corresponding to the younger age. Delay towards damage (3.20). The pattern corresponding to the younger age is impoverished in a motivational and technical way and there are monotonous repetitions of the simplest elements of the pattern. Delay towards disintegration (2.15). The pattern corresponding to the younger age disintegrates into incoherent chaotic fragments.

Distortion (3.00): Premature "breakthrough" (signs of conflict) is followed by unstable pattern use. Distortion towards delay (3.40): The pattern emerges late but develops in a peculiar and uneven way: more complex pattern elements appear before the basic ones. Distortion towards damage (2.50): Intense conflict, a new achievement "falls" into the latent state, i.e. the child abandons the practice. Distortion towards disintegration (1.90): Behavioral orientation is distorted to such an extent that the pattern completely loses its adaptive value and there is confusion among different types of behavior which leads to self-mutilation and traumas. Damage (2.00): Damage towards delay (2.75): The pattern appears unexpectedly with a significant delay (of several years sometimes) against a manic background (the child laughs uncontrollably). Damage towards distortion (2.25): Regression after a period of good mastery of the pattern, which is accompanied by deformation of the pattern, exaggeration of its individual elements. Damage towards disintegration (1.50): Deformation of a pattern with progressive loss of its elements.

Disintegration (1.00): Loss of pattern. Disintegration towards delay (2.75): After losing the pattern, the first signs of it appear as if its development started from scratch. Disintegration towards distortion (1.75): After losing the pattern, it appears in distorted forms. Disintegration towards damage (1.25): After losing the pattern, its deformed elements appear. Absence (0.00).

\section{Assessment procedure}

1) The method implies conducting 2-3 participant observations each lasting 1-1.5 hours, with trial therapeutic interventions with the child, in the presence of the mother (parents). The observation synthesizes the ethological and psychoanalytic approaches (Bardyshevskaya, 1998). The ethological approach consists in the fact that affective-meaningful details and patterns of the child's behavior and his or her interaction with the mother (parents) and a psychologist are recorded on a camera by a specially trained operator and, if possible, are fully monitored by the psychologist. The operator shows a neutral interest in the child but is mainly silent. The psychoanalytic approach consists in the fact that the psychologist gives trial interpretations of changes in the child's current emotional state in connection with certain observed affective loads (external and internal - memories or feelings). Interpretations and comments are given in an imaginative language that the child understands (with the help of toys, drawings, non-verbal communication, speech, it is important that the psychologist uses several formats during one observation). A sign of correct interpretation and optimal selection of affective loads is a safe (without emotional breakdowns exhausting the child and the subsequent shift to a mode of psychophysiological survival during and/or after the examination) change of the child's mode of activity, as well as a consistent change to more highly organized ABCs. The psychologist immediately refuses to try to involve the child in contact if the load used is intolerable (the child hides and leave contact or is fixed on the simplest forms of symbiotic contact). 2) A protocol is made after the observation is completed: the phenomena shall be described in the sequence in which they were observed during the observation session. The protocol (and/or video recording) shall then be analysed by the expert group. For better understanding of what is happening, the psychologist and the operator preliminarily "cut" the video recording into fragments lasting from 1 to several minutes based on the dominant $\mathrm{ABC}$. The sequence of changes in the ABC can be presented schematically to see the "positive" and "negative" episodes. 3) Each noticeable phenomenon shall be referred to a certain ABC. The vector of development of each such phenomenon is identified. To estimate the belonging of a pattern of behavior to this or that $\mathrm{ABC}$, tables are used where typical (archaic, well-recognizable, specific) manifestations of emotional states, behavior and symbolic activity are grouped according to the ABCs - the emphasis is placed on the normal variants with the indication of typical deviations (Bardyshevskaya, 2018). For an estimation of a vector of development, qualitative descriptions and the score menu of estimations are used and also dominant types of connections which are established by the child with other people, toys and objects in a play room are defined. Scores are given for each of the 14 types of behaviour in the 5 ABCs. 4) Recommendations are offered which include the necessary changes in quality, total volume and intensity of mental stress (affective loads), optimal proportions of rigid/flexible structure of training, types of psychotherapy and conditions of socialization that will help to approach the norm.

\section{An example of using a method of the ABC. The types of affective-behavioral development in children 2-3 years old with ASD and PDD}

As an examle, a brief description of a study of psychic development types in children with ASD and PDD using the ABC method is offered. The aim of the study is to identify groups of children according to the similarity of the $\mathrm{ABC}$ profiles (the predominant $\mathrm{ABC}$, vector of development). Besides, the analysis of early development data on early hazards was done to compare the subjective perception of 
these hazards by the mother and objective indicators (number, intensity, acuteness, time and duration of their impact). The hazards reported by the mother to the psychologist during the diagnostic examination and the hazards reported tby her to the psychiatrist on admission to hospital were taken into account. In our work with families we use knowledge of the child's ABC profile while taking into accout the psychological factors that mediate the influence of objective hazards on the child's development.

\subsection{Description of the patients observed}

In studies of children with autism and pervasive developmental disorders, the age of 2-3 years (the period of the "I will do it myself" crisis described by Vygotsky) is key. It is at this age that ASD is increasingly being diagnosed. The very fact of the diagnosis for many parents of children with ASD is a severe trauma. The age period from 2 to 5 years is traditionally considered to be the period of the highest prevalence of early childhood autism syndrome. Identification of early mental development types and options in autism is critical for a scientifically based selection of therapy for the entire preschool period of development, as well as for evaluating its effectiveness. The hazards that had an impact at the time of conception (and even before it), in the intrauterine period of development, during delivery and in the postpartum period, throughout the pre-verbal period of development, are still vividly represented in the memories of parents of at this age and they can be quite accurately "measured". The pathogenic effect of these hazards is detected exactly at the age of 2-3 years old, when the child does not have enough energy resources to switch to independent activity and to reconstruct behavior on the base of speech. At the same time, fears of development and refuse of activity can be fixed for a long time. Finally, at the age of 2-3 years old, the potential of sensomotor preverbal behaviour development reaches its peak. The behavioral repertoire presents a maximum of archaic, well-recognizable, species-specific non-verbal patterns.

The study included 40 2-3 year old children with ASD and PDD who were hospitalized with a parent in the "Mother and Child" department of the Center for Mental Health named after G.E. Sukhareva.

\subsection{Study results}

The variants of affective-behavioral development of children with ADS and PDD are outlined.

Variant 1. Children "on the verge of survival" (4 children, 10\%). Predominant $\mathrm{ABC}$ is the psychophysiological surviving $\mathrm{ABC}$ (serious disruptions of the vital types of behavior, threatening the life of the child, are registered), development of the ABC of symbiotic type is limited by attraction to the inanimate (autoaggression in response to changes in intensity of any stimuli is expressed). Mental development of children fluctuates between damage and distortion (breakdowns of some types of behavior are combined with asynchronous development of other types). Mothers report a large number of pathogenic factors (interrupted pregnancies and miscarriages, the threat of miscarriage almost throughout the whole pregnancy, infections, intoxication, antibiotic treatment during pregnancy, caesarean section, general anaesthesia surgeries, intrusive medical manipulations in the early infancy) and considers the child seriously ill. There is a negative effect of early diagnosis (early stigmatization) and depression of the mother. With properly selected affective loads, the child establishes contact and shows elementary symbolic activity, albeit occasionally and in an unstable way, already at the first session. The mother is not happy with the small advances observed in the child's behaviour during the diagnostic session, considering them a coincidence. Paradoxically, the mother ignores the most important positive changes for development of her child: episodes of clear understanding of the conversational speech, attempts to repeat words by the child in contact with the psychologist (she is sure that the child "does not understand the conversational speech"). Mothers express frustration at the restricted possibilities of modern medicine to cure a child with autism, feel helplessness and expect only a miracle.

The use of "positive" (showing progress in development) videos of diagnostic sessions is promising for changing the image of a child in the mother's mind (from a seriously ill to a developing one). A selection of episodes with signs of playful-dialogic and attachment $A B C$ and the role-imaginative $\mathrm{ABC}$ helps the mother to see the child's potential for the development which she "does not see" with the naked eye.

Variant 2. Children with the predominance of a disturbed ABC of symbiotic type (20 children, $50 \%$ ) "stick" to the primitive sensomotor aspects of the experience, fixing themselves on the reproduction of certain inner feelings (16 children, 40\%). A small proportion (4 children, 10 per cent) consists of children with fixation on abstract sequences (numbers, letters) and stereotyped ordering of objects according to formal attributes (color, size, shape, quantity) with oppression of vital needs (lack of interest in food). The amplitude of developmental fluctuations is wider: between damage and delay. Mothers note a stop in development at 6 months - 2 years of age, linking it to the effects of episodes of injuries, intoxications, infections, including severe complications that coincided with vaccination. In children with fixation on primitive sensorimotor self-stimulation and with auto-aggression in response to the change of stereotype, the contribution of episodes of physical abuse, early sexual trauma, severe emotional deprivation (child rejection) was most significant. Mothers accurately refer to the age of development failure or regression. Mothers report criminal cases of violence by the father. At a diagnostic session, 
they, on the one hand, encourage the child's sexual behaviour and overstimulate him or her. At the same time, they inhibit the child's mental activity by repeating in an authoritarian way that the child wants to sleep and that he or she is not interested or wants to leave. In mothers of children fixed on primitive autostimulation one may notice rent attitudes, fear of cancellation of the diagnosis. In order to improve the situation in the family, it is important to involve the social care professionals, using flexible therapeutic observations in families.

The mothers of "symbiotic" children fixed on elements of abstract systems suffered from depression associated with conflict with their husband and forced relocation. Therefore, the father takes over the care of the child and a symbiotic relationship was formed between him and the child.

The elements of the playful-dialogic and attachment $\mathrm{ABC}$ and the role-imaginative one (initially represented by attempts to make a symbiotic tie with the psychologist and symbolization of symbiotic links) emerge in these children in the second or third session and, especially quickly, if a parent connected with the child by the symbiotic link gradually moves away from the child and leaves the playroom.

Variant 3. Children with difficulties in transitioning from predominant $\mathrm{ABC}$ of symbiotic type to the ABC of expansion (with autistic features and PDD) (8 children, 20\%) whose development oscillates between distortion and delay. In the history there are indications of compromised genetics, older parents, episodes of early infections, injuries, intoxications. It is important that mothers are not fixed on these hazards. They are more concerned about their current relationship with the child. Mothers willingly cooperate with the psychologist, realize the limitations of fixation at the symbiotic stage of development, ask for the psychologist's help in getting out of symbiosis, and involve the father in working with the psychologist. In the conditions of psychotherapeutic consultations, children quickly advance in development of non-verbal communication, food behavior and self-service, active exploration, territorial behavior and constructive play. However, due to a delay in the development of conversational speech, art (depictive) behavior (drawing), the imbalance of development (distortion) is preserved: full understanding of speech when not using it; easy regression to symbiotic forms of contact with the mother.

\subsection{Conclusions}

1. There is a great variety of individual profiles, options and suboptions of affective behavioral development in childhood which can be discerned with the help of the level ABC development assessment. This article describes the variants of psychic development of 2-3 year old children with ASD and PDD. 2. The main purpose of psychotherapy for children with ASD and PDD in the model of level $\mathrm{ABC}$ development is to detect and activate the development of nascent forms of highly organized ABCs rather than fixing the child in the mode of survival and stereotypes. In psychotherapy, the minimum task is to replace the variant of development within the group with a more favorable one; the most ambitious task is to transfer the child to a group with less severity, closer to the norm. 3. There is a correlation between the predominant $\mathrm{ABC}$ and the number of early hazards. If there is a high concentration of hazards, their prolonged impact on the fetus and/or the baby, and the mother's strong psychological stress during pregnancy, childbirth and the postnatal period, the development of the child's behavior is fixated on the basic ABCs. The specifics of parental perception of the contribution of these objective hazards vary among children from different groups in terms of the severity of autism. These differences should be taken into account when choosing how to work with the families.

\section{References}

Bardyshevskaya, М. К. (2012). Уровневая модель развития аффективно-поведенческих комплексов. [Level model of affective-behavioral complexes development]. Issues of Psychology, 3, 68-77.

Bardyshevskaya, М. К. (2018). Аффект, поведение и символическая активность: методика наблюдения и диагностики. [Affect, behavior and symbolic activity: methods of observation and diagnostics]. Moscow, Russia: Moscow Department of Public Health, Scientific and Practical Center for Mental Health of Children and Adolescents named after G. E. Sukhareva.

Bardyshevskaya, M. K., Lebedinsky V. V. (2003). Диагностика эмоциональных нарушений у детей. [Assessment of childrens' emotional disorders]. Moscow, Russia: "Psychology" training center.

Bardyshevsky, M. (1998). The compensation of autistic features during a little boy's second year: overcoming pain through the development of attachment. The International Journal of Infant Observation, 2(1), 40-57.

Bernstein, N. A. (1967). The co-ordination and regulation of movements. Oxford: Pergamon Press.

Lebedinsky, V. V. (1985). Психические нарушения у детей. [Mental disorders in children]. Moscow, Russia: Lomonosov Moscow State University Publishing House.

Lebedinsky, V. V., Nikolskaya O. S., Baenskaya E. R., Libling M. M. (1990). Эмоциональные нарушения у детей и их коррекция. [Childrens' emotional disorders and its correction]. Moscow, Russia: Lomonosov Moscow State University Publishing House.

Sukhareva, G. E. (1959). Лекции по детской психиатрии в 3 томах, том 3. [Lectures on child psychiatry in 3 volumes, vol. 3]. Moscow, Russia: Medgiz. 